\title{
ТОЧКИ СОПРИКОСНОВЕНИЯ: МАТЕМАТИЧЕСКОЕ ПРОГРАММИРОВАНИЕ И МЕНЕДЖМЕНТ. ОБЗОР
}

Стороженко В.Л., Ильичева О.А. ФГБОУ ВО «Донской государственный технический университет», г. Ростов-на-Дону, Россия

Математическое программирование в настоящее время применяется в совершенно разных направлениях. В статье будет дан обзор влияния $u$ использования математического программирования в менеджменте. Поймем насколько большую роль играет программирование в науке управления.

Ключевые слова: математическое программирование, менеджмент, моделирование, симплекс-метод, задача оптимального распределения, оптимизация, линейное программирование.

В XXI веке немалая часть наук так или иначе связаны с математическим программированием. Наука управления (менеджмент) не является исключением. Менеджмент характеризуется научным подходом к принятию управленческих решений. Хотя его корни можно проследить до проблем, возникших в ранних цивилизациях, лишь во время Второй мировой войны он стал определяться как респектабельный и четко определенный массив знаний. C тех пор он рос впечатляющими темпами, беспрецедентный для большинства научных достижений; это меняет наше отношение к принятию решений и проникает во все мыслимые области применения, охватывая широкий спектр проблем бизнеса, промышленности, армии и государственного сектора.

Менеджмент был известен под множеством других имен. Некоторые люди склонны идентифицировать научный подход к решению управленческих проблем под такими другими названиями, как системный анализ, анализ затрат и выгод и анализ эффективности затрат.

Математическое программирование, и особенно линейное программирование, является одной из наиболее развитых и наиболее используемых отраслей менеджмента. Это касается оптимального распределения ограниченных ресурсов между конкурирующими видами деятельности в соответствии с рядом ограничений, налагаемых характером изучаемой проблемы. Эти ограничения могут отражать финансовые, технологические, маркетинговые, организационные или многие другие соображения.

В общих чертах, математическое программирование (в данном контексте) может быть определено как математическое представление, направленное на программирование или планирование наилучшего возможного распределения ограниченных ресурсов. Когда математическое представление использует 
исключительно линейные функции, мы имеем модель линейного программирования.

В 1947 году Джордж Б. Данциг, в то время входивший в исследовательскую группу ВВС США, известную как Project SCOOP (Научное вычисление оптимальных программ), разработал симплекс-метод для решения общей задачи линейного программирования. Исключительная вычислительная эффективность и надежность симплекс-метода, a также доступность высокоскоростных цифровых компьютеров сделали линейное программирование самым мощным из когда-либо разработанных методов оптимизации и наиболее широко применяемым в бизнес-среде.

Поскольку математическое программирование является лишь инструментом широкой дисциплины, известной как менеджмент, сначала необходимо понять подход науки управления и определить роль математического программирования в этом подходе.

Трудно дать однозначное определение менеджмента. Даная область извлекает выгоду из вкладов, происходящих в социальных и естественных науках, эконометрике и математике, многие из которых избегают жесткости определения.

Менеджмент характеризуется использованием математических моделей в предоставлении руководящих указаний руководителям для принятия эффективных решений в состоянии текущей информации или в поиске дополнительной информации, если текущих знаний недостаточно для принятия правильного решения.

Есть несколько элементов этого утверждения, которые заслуживают особого внимания. Во-первых, сущностью науки управления является подход к построению модели, то есть попытка охватить наиболее значимые особенности рассматриваемого решения с помощью математической абстракции. В то же время они должны обеспечивать полное и реалистичное представление среды принятия решений, включая все элементы, необходимые для характеристики сущности изучаемой проблемы. Это непростая задача, но, если все сделано правильно, она предоставит огромный инструмент для использования в сложных ситуациях принятия решений.

Во-вторых, посредством этих усилий по разработке модели менеджмент пытается дать указания руководителям или, другими словами, улучшить понимание руководителями последствий своих действий. Цель состоит в том, чтобы поддержать управленческие действия. Комплементарность между превосходными вычислительными возможностями, обеспечиваемыми моделью, и более высокими оценочными способностями человека, принимающего решения, является ключом к успешному научному подходу в управление.

Наконец, именно сложность исследуемого решения, а не инструмент, используемый для исследования процесса принятия решений, должна определять объем информации, необходимой для эффективного принятия этого решения. Модели подвергались критике за создание необоснованных требований к информации. На самом деле в этом нет необходимости. Наоборот, 
модели могут быть построены в текущем состоянии доступной информации, и их можно использовать для оценки того, является ли экономически желательным сбор дополнительной информации.

Научная литература включает несколько подходов в классификации моделей. Сначала будет описана категоризация, которая идентифицирует широкие типы моделей в соответствии со степенью реализма, которого они достигают при представлении данной проблемы:

1. Первый тип модели является оперативным упражнением. Этот подход к моделированию напрямую связан с реальной средой, в которой будет приниматься решение. Усилия по моделированию просто включают в себя разработку серии экспериментов, которые будут проводиться в этой среде, а также измерение и интерпретацию результатов этих экспериментов. Предположим, например, что мы хотели бы определить, какую смесь нескольких видов сырой нефти следует смешивать на нефтеперерабатывающем заводе, чтобы наиболее эффективным образом удовлетворить рыночные требования к конечным продуктам, которые будут поставляться с этого завода. Если бы нам нужно было провести оперативное учение в поддержку решения, мы бы попробовали разные количества нескольких комбинаций типов сырой нефти непосредственно в фактическом процессе переработки и наблюдали бы полученные доходы и расходы, связанные с каждым альтернативным сочетанием. Проведя довольно много испытаний, мы начнем понимать взаимосвязь между вводом сырой нефти и чистым доходом, полученным в процессе переработки, что поможет нам определить подходящее сочетание.

Чтобы этот подход работал успешно, необходимо тщательно спланировать эксперименты, оценить результаты эксперимента в свете ошибок, которые могут быть вызваны неточностями измерений, и сделать выводы о принятых решениях на основе ограниченного количество выполненных наблюдений. Суть оперативного упражнения - это индуктивный процесс обучения, характерный для эмпирических исследований в естественных науках, в котором обобщения извлекаются из конкретных наблюдений за данным явлением.

Операционные упражнения содержат высочайшую степень реалистичности любого вида подхода к моделированию, поскольку едва ли вводятся какие-либо внешние абстракции или упрощения, кроме тех, которые связаны с интерпретацией наблюдаемых результатов и обобщениями, которые следует извлечь из них. Тем не менее, способ чрезвычайно, как правило, непомерно дорогой для реализации. Более того, в большинстве случаев невозможно провести исчерпывающий анализ альтернатив, доступных для лица, принимающего решение. Это может привести к серьезной субоптимизации в окончательных выводах.

2. Второй тип модели в этой классификации - игровые. В этом случае строится модель, которая является абстрактным и упрощенным представлением реальной среды. Эта модель обеспечивает механизм реагирования для оценки эффективности предложенных альтернатив, которые лицо, принимающее решения, должно предоставлять организованным и последовательным образом. 
Модель - это просто устройство, которое позволяет лицу, принимающему решение, протестировать производительность различных альтернатив, которые, по-видимому, стоят того, чтобы их использовать. Кроме того, в игровой ситуации всем людям, которые влияют на среду принятия решений, разрешается принимать активное участие, предоставляя материалы, где они обычно отвечают за фактическую реализацию своей деятельности. Если в нашем предыдущем примере используется игровой подход, процесс переработки будет представлен компьютерной или математической моделью, которая может принять любую структуру.

Модель должна с приемлемой степенью точности отражать взаимосвязи между входами и выходами процесса переработки. Впоследствии весь персонал, который участвует в структурировании процесса принятия решений, будет иметь возможность взаимодействовать с моделью. Руководитель производства будет составлять производственные планы, менеджер по маркетингу будет заключать контракты и разрабатывать маркетинговые стратегии, менеджер по закупкам будет определять цены и источники сырой нефти и разрабатывать программы закупок и так далее. Конечно, мы потеряем некоторую степень реализма в нашем подходе к моделированию в отношении оперативных упражнений, поскольку мы работаем с абстрактной средой, но мы сохранили некоторые человеческие взаимодействия реального процесса. Однако стоимость обработки каждой альтернативы была уменьшена, а скорость измерения производительности - увеличена.

Игры используются в основном в качестве обучающего устройства для развития понимания тех сложностей, которые присущи процессу принятия решений.

3. Имитационные модели похожи на игровые модели, за исключением того, что все лица, принимающие решения, исключаются из процесса моделирования. Модель предоставляет средства для оценки эффективности ряда альтернатив, предоставляемых извне лицом, принимающим решения, без учета взаимодействия человека на промежуточных этапах расчета модели.

Подобно операционным упражнениям и играм, имитационные модели не генерируют альтернативы и не дают оптимального ответа на изучаемое решение. Эти типы моделей носят индуктивный и эмпирический характер; они полезны только для оценки эффективности альтернатив, определенных ранее лицом, принимающим решения

Если привести имитационную модель в примере с нефтеперерабатывающим заводом, то заранее запрограммировали бы большое количество комбинаций количеств и типов сырой нефти, и получили бы чистую прибыль, связанную с каждой альтернативой, без каких-либо внешних затрат лица, принимающие решения. После того, как были бы получены результаты модели, можно было проводить новые эксперименты, пока не почувствовали, что достигли правильного понимания проблемы.

Многие имитационные модели принимают форму компьютерных программ, в которых логические арифметические операции выполняются в 
заранее определенной последовательности. Поэтому нет необходимости определять проблему исключительно в аналитических терминах.

4. Четвертая модельная категория является аналитической моделью. В этом типе модели проблема представляется полностью в математических терминах. Модель вычисляет оптимальное решение, то есть такое, которое удовлетворяет всем ограничениям и дает наилучшее возможное значение целевой функции

В примере с нефтеперерабатывающим заводом использование аналитической модели подразумевает постановку в качестве цели максимизации чистого дохода, полученного от работы на нефтеперерабатывающем заводе, в зависимости от типов и количества используемой сырой нефти. Решением модели будет точное количество каждого доступного типа сырой нефти, которое будет обработано, что максимизирует чистую прибыль в рамках предложенного набора ограничений.

В последние два десятилетия линейное программирование было неоспоримой аналитической моделью, используемой для решения подобных задач. Аналитические модели обычно являются наименее дорогими и простыми в разработке моделями. Тем не менее, они вводят самую высокую степень упрощения в представлении модели.

Для получения общей формулировки проблемы математического программирования, необходимо начать с формального представления общей модели линейного программирования.

В математических терминах модель линейного программирования может быть выражена как максимизация (или минимизация) целевой функции с учетом заданного набора линейных ограничений. В частности, задача линейного программирования может быть описана как нахождение значений $n$ решающих переменных $x_{1}, x_{2}, \ldots, x_{n}$, так что они максимизируют (или минимизируют) целевую функцию $Z$, где

$$
Z=c_{1} x_{1}+c_{2} x_{2}+\cdots+c_{n} x_{n} \rightarrow \max
$$

при условии соблюдения следующих ограничений:

$$
\begin{aligned}
& a_{11} x_{1}+a_{12} x_{2}+\cdots+a_{1 n} x_{n} \leq b_{1}, \\
& a_{21} x_{1}+a_{22} x_{2}+\cdots+a_{2 n} x_{n} \leq b_{2}, \\
& \vdots \\
& a_{m 1} x_{1}+a_{m 2} x_{2}+\cdots+a_{m n} x_{n} \leq b_{m} \\
& \text { где, как правило: } \quad x_{1} \geq 0, x_{2} \geq 0, \cdots, x_{n} \geq 0
\end{aligned}
$$

а $c_{j}, a_{i j}$ и $b_{i}$ постоянные.

Легко дать интерпретацию общей задаче линейного программирования, только что сформулированной в терминах производственной задачи. Например, мы могли бы предположить, что на данном производственном объекте нет $n$ возможных продуктов, которые мы можем производить; для каждого из них мы хотим определить уровень производства, который мы обозначим через 
$x_{1}, x_{2}, \ldots, x_{n}$. Кроме того, эти продукты конкурируют за $m$ ограниченных ресурсов, которые могут быть доступной рабочей силой, мощностями машин, спросом на продукцию, оборотным капиталом и т. д., И обозначены как $b_{1}, b_{2}, \ldots, b_{m}$. Пусть $a_{i j}$ будет количеством ресурса $i$ (где $i$ принимает значение от 1 до $\mathrm{m}$ ), которое требуется для продукта $j$ (где ј принимает значение от 1 до n), и пусть $c_{j}$ будет единицей прибыли продукта $j$. Затем модель линейного программирования стремится определить объем производства каждого продукта таким образом, чтобы максимизировать общую результирующую прибыль z (1), учитывая, что доступные ресурсы не должны быть превышены (2), и что мы может производить только положительные или нулевые количества каждого продукта (3).

Линейное программирование не ограничивается структурой задачи, представленной выше. Во-первых, вполне возможно минимизировать, а не максимизировать целевую функцию. Кроме того, ограничения «больше или равно» или «равно» могут обрабатываться одновременно с ограничениями «меньше или равно» (представленными в ограничениях 2). Наконец, некоторые переменные могут принимать как положительные, так и отрицательные значения.

Существует некоторая техническая терминология, связанная с математическим программированием, которую необходимо определить в более точных терминах. Множество всех значений переменных решения, характеризуемых ограничениями (2) и (3), образуют допустимую область рассматриваемой задачи. Возможное решение, которое дополнительно оптимизирует целевую функцию (1), называется оптимально возможным решением.

Решение линейной программы может привести к трем возможным ситуациям:

1. Линейная программа может быть неосуществимой, что означает отсутствие значений переменных решения $x_{1}, x_{2}, \ldots, x_{n}$, которые одновременно удовлетворяют всем ограничениям (2) и (3).

2. Оно может иметь неограниченное решение, означающее, что, если мы максимизируем, значение целевой функции может быть увеличено на неопределенный срок без нарушения каких-либо ограничений. (Если мы минимизируем, значение целевой функции может уменьшаться до бесконечности.).

3. В большинстве случаев у него будет хотя бы одно конечное оптимальное решение, и часто будет несколько оптимальных решений.

Симплексный метод решения линейных программ обеспечивает эффективную процедуру для построения оптимального решения, если оно существует, или для определения, является ли задача неосуществимой или неограниченной.

Необходимо обратить внимание, что в формулировке линейного программирования переменные решения могут принимать любое непрерывное значение. Например, такие значения, что $x_{i}$ будет принимать вещественные 
значения являются совершенно приемлемыми, если они удовлетворяют ограничениям (2) и (3). Важным расширением этой модели линейного программирования является требование, чтобы все или некоторые переменные решения были ограничены целочисленными значениями.

Другое фундаментальное расширение вышеупомянутой модели состоит в том, чтобы позволить целевой функции, или ограничениям, или обоим, быть нелинейными функциями. Общая модель нелинейного программирования может быть сформулирована как нахождение значений переменных решения $x_{1}, x_{2}, \ldots, x_{n}$, которые максимизируют (или минимизируют) целевую функцию $Z$, где

$$
Z=f\left(x_{1}, x_{2}, \ldots, x_{n}\right) \rightarrow \max
$$

при условии соблюдения следующих ограничений:

$$
\begin{aligned}
& f_{1}\left(x_{1}, x_{2}, \ldots, x_{n}\right) \leq b_{1} \\
& f_{2}\left(x_{1}, x_{2}, \ldots, x_{n}\right) \leq b_{2} \\
& f_{m}\left(x_{1}, x_{2}, \ldots, x_{n}\right) \leq b_{m}
\end{aligned}
$$$$
\vdots
$$

где:

$$
x_{1} \geq 0, x_{2} \geq 0, \cdots, x_{n} \geq 0
$$

Также стоит отметить, что при максимизации целевой функции, неравенства записываются со знаком «меньше или равно», а при минимизации целевой функции - со знаком «больше или равно».

Часто в нелинейном программировании значения правой части включаются в определение функции $f_{i}\left(x_{1}, x_{2}, \ldots, x_{n}\right)$, оставляя правую часть равной нулю. Чтобы решить проблему нелинейного программирования, необходимо сделать некоторые предположения о форме и поведении задействованных функций. Необходимо, чтобы нелинейные функции были достаточно хорошими, чтобы иметь вычислительно эффективные средства для поиска решения.

Модели оптимизации могут подчиняться различным классификациям в зависимости от принятой нами точки зрения. В соответствии с количеством периодов времени, рассматриваемых в модели, модели оптимизации можно классифицировать как статические (один период времени) или многоступенчатые (несколько периодов времени).

Динамическое программирование является одним из подходов к решению многоступенчатых задач. Кроме того, в настоящее время в области крупномасштабного линейного программирования ведется значительная исследовательская работа по разработке специальных алгоритмов для решения многоступенчатых задач.

Другой важный способ классификации моделей оптимизации относится к поведению параметров модели. Если параметры являются известными константами, модель оптимизации называется детерминированной. Если параметры указаны как неопределенные величины, значения которых характеризуются распределениями вероятностей, то модель оптимизации 
называется стохастической. Наконец, если некоторым параметрам разрешено систематически изменяться и определяются изменения в оптимальном решении, соответствующие изменениям в этих параметрах, оптимизационная модель называется параметрической. В целом, стохастическое и параметрическое математическое программирование порождает гораздо более сложные проблемы, чем детерминированное математическое программирование. Детерминированное линейное программирование может эффективно применяться для решения очень больших задач, содержащих до 5000 строк и практически неограниченное количество переменных. Кроме того, в линейном программировании анализ чувствительности и параметрическое программирование могут быть эффективно проведены после получения детерминированного оптимального решения.

Третий способ классификации моделей оптимизации связан с поведением переменных в оптимальном решении. Если переменным разрешено принимать любое значение, которое удовлетворяет ограничениям, модель оптимизации называется непрерывной. Если переменным разрешено принимать только дискретные значения, модель оптимизации называется целочисленной или дискретной. Наконец, когда в задаче есть некоторые целочисленные переменные и некоторые непрерывные переменные, модель оптимизации называется смешанной.

В общем, проблемы с целочисленными переменными значительно сложнее решить, чем с непрерывными переменными. Сетевые модели представляют собой класс моделей линейного программирования, которые являются исключением из этого правила, поскольку их специальная структура приводит к целочисленным оптимальным решениям. Хотя значительный прогресс был достигнут в общей области смешанного и целочисленного линейного программирования, до сих пор не существует алгоритма, который мог бы эффективно решать все общие целочисленные линейные программы среднего размера за разумное время, хотя для особых задач адекватные вычислительные методы были разработаны.

\section{Список литературы}

1. Мазалов В.В., Чиркова Ю.В., Сетевые игры: Учебное пособие. - СПб.: Издательство «Лань», 2018. - 320 с.: ил. - (Учебники для вузов. Специальная литература), ISBN 978-5-8114-3035-2.

2. Москвитин А.А., Решение задач на компьютерах. Спецификация задачи: Учебное пособие. - СПб.: Издательство «Лань», 2018. - 228 с.: ил. (Учебники для вузов. Специальная литература), ISBN 978-5-8114-3095-6.

3. Горбач Б.А., Шахов В.Г., Математическое моделирование. Построение моделей и численная реализация: Учебное пособие. - СПб.: Издательство «Лань», 2018. - 292 с.: ил. - (Учебники для вузов. Специальная литература), ISBN 978-5-8114-2168-8. 